\title{
Transitional Care Interventions for Patients with Heart Failure: An Integrative Review
}

\author{
Hai Mai Ba ${ }^{1} \mathbb{C}$, Youn-Jung Son ${ }^{2}$, Kyounghoon Lee ${ }^{3,4}$ and Bo-Hwan Kim ${ }^{4,5, *(\mathbb{C})}$ \\ 1 Department of Nursing, Gachon University Graduate School, Incheon 21936, Korea; \\ maibahai211@gmail.com \\ 2 Red Cross College of Nursing, Chung-Ang University, Seoul 06974, Korea; yjson@cau.ac.kr \\ 3 College of Medicine, Division of Cardiology, Gachon University, Incheon 21565, Korea; \\ cardioman@gilhospital.com \\ 4 Cardiovascular Research Institute, Gachon University, Incheon 21565, Korea \\ 5 College of Nursing, Gachon University, Incheon 21936, Korea \\ * Correspondence: bhkim@gachon.ac.kr; Tel.: +82-32-820-4213
}

Received: 28 February 2020; Accepted: 17 April 2020; Published: 23 April 2020

\begin{abstract}
Heart failure (HF) is a life-limiting illness and presents as a gradual functional decline with intermittent episodes of acute deterioration and some recovery. In addition, HF often occurs in conjunction with other chronic diseases, resulting in complex comorbidities. Hospital readmissions for $\mathrm{HF}$, including emergency department (ED) visits, are considered preventable. Majority of the patients with HF are often discharged early in the recovery period with inadequate self-care instructions. To address these issues, transitional care interventions have been implemented with the common objective of reducing the rate of hospital readmission, including ED visits. However, there is a lack of evidence regarding the benefits and adverse effects of transitional care interventions on clinical outcomes and patient-related outcomes of patients with HF. This integrative review aims to identify the components of transitional care interventions and the effectiveness of these interventions in improving health outcomes of patients with HF. Five databases were searched from January 2000 to December 2019, and 25 articles were included.
\end{abstract}

Keywords: heart failure; integrative review; transitional care intervention

\section{Introduction}

Heart failure (HF) is a life-threatening syndrome in which the cardiac pump does not sufficiently maintain the blood flow to meet the body's needs for oxygen and blood [1]. HF syndrome constitutes a major global health problem, affecting at least 26 million people worldwide [2,3]. In addition, the prevalence of HF will dramatically increase with an aging population. In the United States, there are currently 5.7 million cases of $\mathrm{HF}$, with the projected annual incidence expected to exceed 8 million by 2030 [4]. Likewise, the prevalence of patients with HF in Korea was estimated to be $1.53 \%$ in 2013 and is expected to increase 2.2 -fold to $3.4 \%$ by 2040. In other words, over 1.7 million Koreans are estimated to be affected by HF by 2040 [5].

HF contributes to substantial morbidity, high mortality, frequent readmissions, and emergency department (ED) visits because of progressive HF pathogenesis, poor self-management, and emergent signs and symptoms owing to increased hemodynamic overload [1]. Consequently, these vicious cycles negatively affect patients' quality of life (QoL) [6] and health-related QoL (HRQoL) [7] as well as increase the burden of healthcare cost on patients and their families [8].

Despite advancements in medical procedures and treatments, HF management remains a challenge to healthcare providers. Of the various methods to manage HF, transitional care intervention is the 
most innovative program to improve the continuity of care for patients with HF from admission to after hospital discharge $[9,10]$. A transitional care intervention based on transfer from hospital to home was designed, evaluated, and implemented by Mary Naylor at the University of Pennsylvania in Philadelphia to improve the outcomes of chronic older patients as well as to reduce the costs of healthcare [11]. According to the American Heart Association, transitional care interventions for HF should span the care continuum [10]. Specifically, the most important core features of transitional care are comprehensive in-hospital planning, postdischarge follow-up, and ongoing support via telephone or home visits for chronically ill, high-risk, older patients hospitalized for medical services [10,11].

Until now, there have been some systematic reviews or meta-analyses of randomized controlled trials (RCTs) reporting on transitional care interventions $[9,12,13]$. There have also been reports on the evidenced effectiveness of transitional care interventions based on non-RCT study designs. Integrative reviews combine data of both experimental and non-experimental studies to fully understand the phenomenon being analyzed. In addition, integrative reviews are the best comprehensive methodological approach to evaluate theoretical and empirical literature with a range of purposes, such as analysis of methodological problems related to a topic [14]. Therefore, the specific objectives of this integrative review were to analyze the components of transitional care intervention and examine the effectiveness of these interventions in improving clinical and patient-related outcomes of HF.

\section{Materials and Methods}

\subsection{Study Design}

This integrative literature review studies and summarizes previous research by drawing conclusions from individual studies believed to address the relevant topics. The integrative review methodology described by Whittemore and Knafl [14] was used. This review includes five stages: problem identification, literature search, data evaluation, data analysis, and presentation.

\subsection{Problem Identification}

The central question of this integrative review is "What are the outcomes/effectiveness of transitional care intervention/transitional care programs for HF?" The characteristics evaluated included completeness of the intervention; nature of the intervention (educational alone or including multiple interventions); who was the target population; and what were the outcomes (clinical outcomes: all-cause mortality rates, readmission rates, average number of ED visits per patient, length of stay (LOS) in index care, index care costs, and follow-up costs; patient-related outcomes: QoL, HRQoL, satisfaction, and quality of transition).

\subsection{Literature Search}

We performed a search for relevant articles in the databases of PubMed, Cumulative Index of Nursing and Allied Health Literature, Web of Science, EMBASE, and COHRANE. The keywords used to search were as follows: (1) "transitional care intervention" OR "transitional care program" OR "transition of care" OR "transition of care model" OR "postdischarge follow up" and (2) "HF patients." Articles included in this integrative review met the following criteria: (1) focus on adult HF patients, (2) concern for the effectiveness of transitional care interventions or transitional care programs, (3) clinical and patient-related outcomes mentioned in stage 1 as dependent variables, (4) patients transiting from the hospital to home, (5) accessibility of the full text of articles in detail, (6) published in English, and (7) published between January 2000 and December 2019. Non-original research articles, study protocols, development of instrument, and studies that did not clearly describe the transitional care procedure or the process of intervention were excluded. Two authors (H.M.B. and B.-H.K.) independently screened the studies according to the criteria and discussed the screening results. Finally, 25 papers met the criteria and were included in this review. 


\subsection{Data Evaluation}

The quality of studies with different designs was assessed using the Mixed Methods Appraisal Tool (MMAT; 2018 version), which is a critical tool designed for the appraisal stage of systematic mixed methods studies, including integrative reviews [15]. MMAT enables the appraisal of the methodological quality based on the following five categories of studies: qualitative research, RCTs, non-randomized studies, quantitative descriptive studies, and mixed methods studies. Computing the score from the grading of each item is discouraged. Instead of computing the score, a direction to write a more detailed evaluation of each item to better assess the quality of the included studies is used. Therefore, excluding studies with low methodological quality is usually discouraged.

The 25 included studies were evaluated using MMAT. The two screening questions were applied to all reviewed studies regardless of the design. Afterward, the appropriate criteria were selected and answered according to each study design. Two authors (H.M.B. and B.-H.K.) independently evaluated the studies according to the criteria and discussed the results.

All 25 studies satisfied the two screening questions. Of the eight RCTs, seven satisfied all quality appraisal criteria and one showed a higher dropout rate in the control group than in the intervention group, resulting in mortality bias [16]. Of the 15 quantitative non-randomized studies, 13 met all quality appraisal criteria, one showed a rate of complete outcome data of $<80 \%$ [17], and another lacked inclusion and exclusion criteria for participant selection [18]. Of the two quantitative descriptive studies, one satisfied all criteria [19] and the other lacked criteria for the risk of non-response bias; moreover, the authors did not balance for any potential confounding factors such as patient age, level of education, or duration of HF [20].

\subsection{Data Analysis}

We fully read and analyzed each of the 25 selected studies independently. We met regularly for review, data reduction, and data extraction to complete the integrative review. The characteristics and results of the studies are summarized in the order of year in Table 1. The three sections in Table 1 are as follows: (1) general study information (author (year), country, study design, sample size (male \%), type of HF, age, racial ethnicity, and HF severity); (2) major information regarding transitional care interventions (intervention, major intervention provider, transition time/place, and intervention duration); and (3) major outcomes (clinical and patient-related outcomes). The effectiveness of transitional care interventions for patients with HF was analyzed in two domains: clinical outcomes (all-cause or HF readmission rate, ED visit, mortality rate, LOS, and care costs) and patient-related outcomes (QoL, HRQoL, satisfaction, and quality of transition). In addition, the intervention components were analyzed according to previous transitional care models and are summarized in Table 2. These include (1) predischarge and (2) postdischarge interventions. Figure 1 presents a flow diagram of all review stages. 

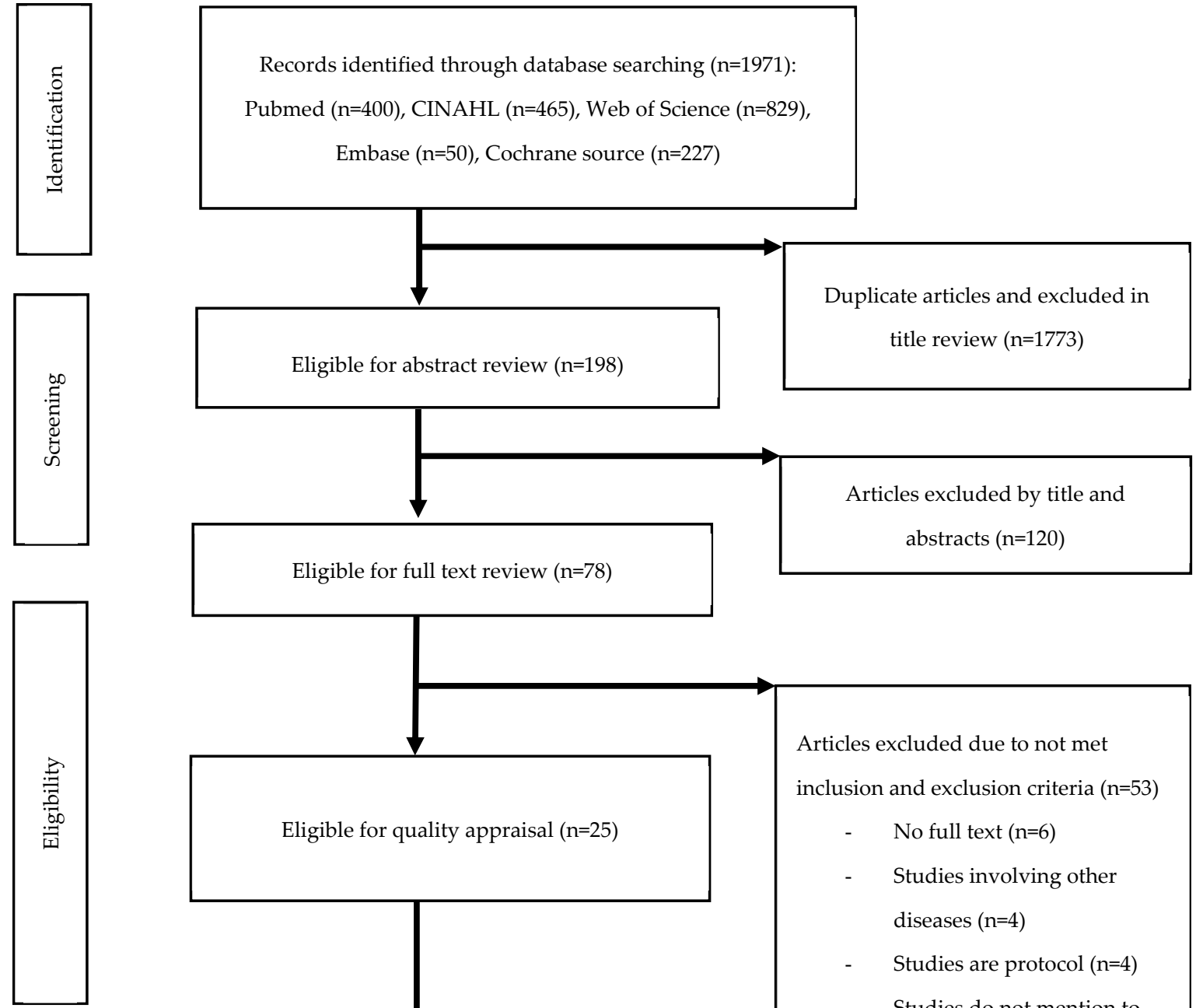

Eligible for full text review $(\mathrm{n}=78)$

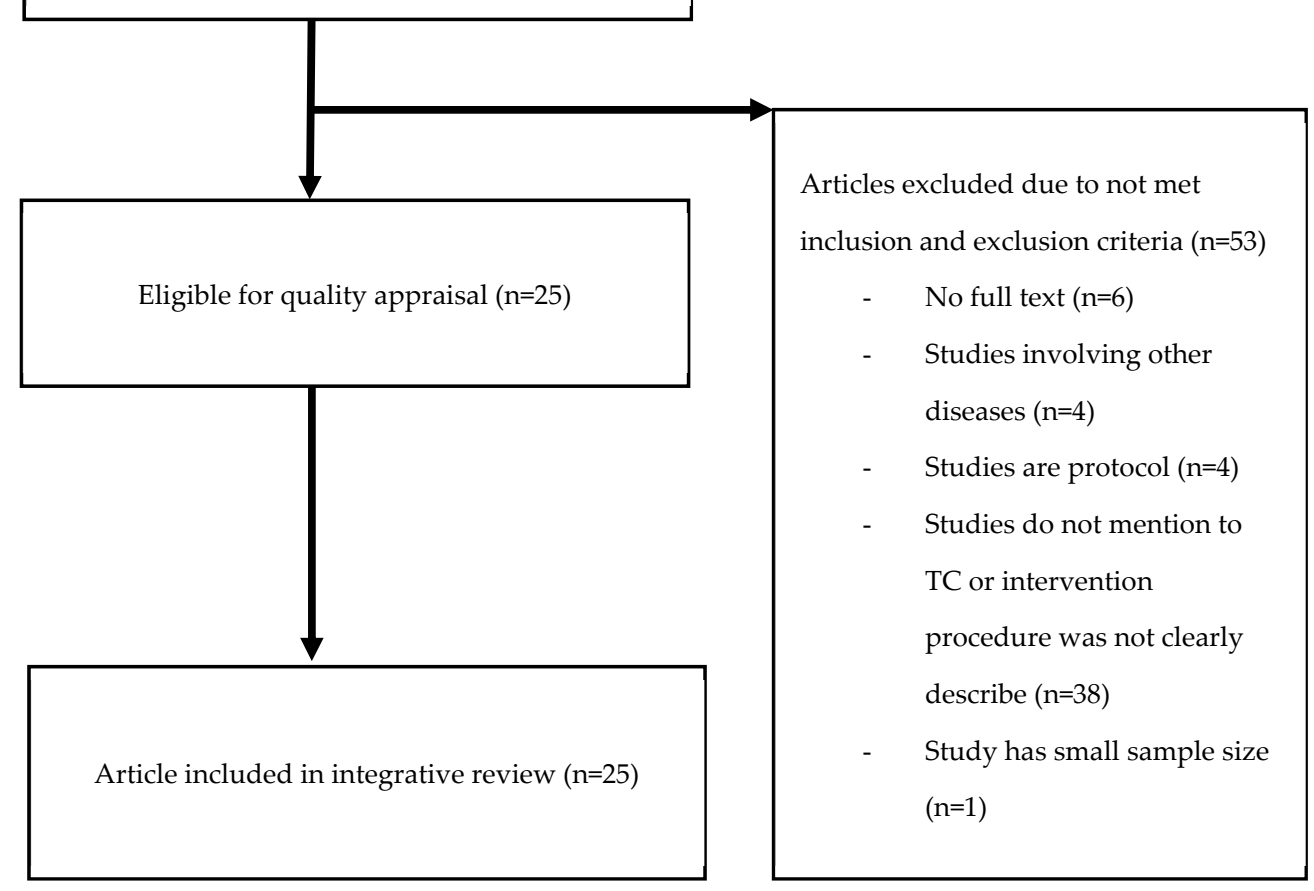

Figure 1. Preferred reporting items for systematic reviews and meta-Analyses (PRISMA) flow diagram outlining the literature search and study selection. 
Table 1. Descriptive summary of transitional care intervention studies for patients with HF $(n=25)$.

\begin{tabular}{|c|c|c|c|c|c|}
\hline \multirow{2}{*}{$\begin{array}{l}\text { Author (Year) } \\
\text { Country }\end{array}$} & \multirow{2}{*}{ Study Design } & \multirow{2}{*}{$\begin{array}{l}\text { Sample (Number (Male \%), } \\
\text { Type of HF, Age, Racial } \\
\text { Ethnicity, HF Severity) }\end{array}$} & \multirow{2}{*}{$\begin{array}{l}\text { Contents of Intervention (Intervention, Major } \\
\text { Intervention Provider, Transition Time/Place, } \\
\text { Intervention Duration) }\end{array}$} & \multicolumn{2}{|c|}{ Outcomes (Significance) } \\
\hline & & & & Clinical Outcomes & Patient-Related Outcomes \\
\hline \multirow{3}{*}{$\begin{array}{l}\text { Harrison, et al. (2002) } \\
\text { [21] Canada }\end{array}$} & \multirow{3}{*}{ RCT (Exp. vs. Con.) } & $\begin{array}{l}\mathrm{N}=192(55 \%) \\
\text { Congestive HF }\end{array}$ & $\begin{array}{c}\text { Transitional care intervention } \\
\text { Nurses }\end{array}$ & Hospital readmissions (-) & HRQoL $(+++)$ \\
\hline & & 75 years & Hospital to home & \multirow{2}{*}{ All-cause emergency room visits (+) } & \multirow[b]{2}{*}{ QoL (-) } \\
\hline & & $\begin{array}{c}\text { N/A } \\
\text { NYHA III/IV 77\% }\end{array}$ & Until 2 weeks after hospital discharge & & \\
\hline \multirow{3}{*}{$\begin{array}{l}\text { Naylor et al. (2004) } \\
\text { [22] UnUSA }\end{array}$} & \multirow{3}{*}{ RCT (Exp. vs. Con.) } & $\begin{array}{c}\mathrm{N}=239(43 \%) \\
\mathrm{HF}\end{array}$ & $\begin{array}{l}\text { Transitional care intervention (APN-directed } \\
\text { discharge planning and home follow-up) }\end{array}$ & \multirow{2}{*}{$\begin{array}{c}\text { First rehospitalization period or death at } 52 \\
\text { weeks }(+)\end{array}$} & \multirow{2}{*}{$\mathrm{QoL}(+)$} \\
\hline & & 76 years & & & \\
\hline & & $\begin{array}{c}\text { African American } 36 \% \\
\operatorname{LVEF}(\sim 45 \%-20 \%) \sim 69 \%-72 \%\end{array}$ & $\begin{array}{l}\text { Hospital to home } \\
3 \text { months }\end{array}$ & $\operatorname{Cost}(+)$ & Satisfaction $(+++)$ \\
\hline \multirow{2}{*}{$\begin{array}{l}\text { Williams et al. (2010) } \\
\text { [23] United Kingdom }\end{array}$} & \multirow{2}{*}{$\begin{array}{l}\text { Quasi-experiment } \\
\text { (Exp. vs. Con.) }\end{array}$} & $\begin{array}{l}\mathrm{N}=97(52 \%) \\
\quad \mathrm{CHF}\end{array}$ & Transitional care service & Readmissions at 30 days $(-)$ & \multirow{2}{*}{$\begin{array}{l}\text { Satisfaction (non-statistics, positive } \\
\text { feedback) }\end{array}$} \\
\hline & & $\begin{array}{l}\text { 71-78 years } \\
\text { N/A } \\
\text { N/A }\end{array}$ & $\begin{array}{l}\text { CNS } \\
\text { Hospital to home } \\
18 \text { weeks }\end{array}$ & $\operatorname{LOS}(-)$ & \\
\hline \multirow{3}{*}{$\begin{array}{l}\text { Barnason et al. (2010) } \\
\text { [24] USA }\end{array}$} & \multirow{3}{*}{ RCT (Exp. vs. Con.) } & $\begin{array}{c}\mathrm{N}=38(65 \%) \\
\mathrm{HF}\end{array}$ & $\begin{array}{l}\text { Hospital transition intervention } \\
\text { Research nurse }\end{array}$ & \multirow{3}{*}{$\mathrm{N} / \mathrm{A}$} & Medication adherence $(+++)$ \\
\hline & & $\begin{array}{l}77 \text { years } \\
\text { N/A }\end{array}$ & Hospital to home & & Self-efficacy for $\mathrm{HF}$ self-care $(+++)$ \\
\hline & & NYHA III 55\% & $2 \sim 3$ weeks & & HRQoL (++) \\
\hline \multirow{4}{*}{$\begin{array}{l}\text { Stauffer et al. (2011) } \\
\text { [25] USA }\end{array}$} & \multirow{4}{*}{$\begin{array}{l}\text { Prospective study } \\
\text { (Exp. vs. Con.) }\end{array}$} & $\mathrm{N}=1025(47 \%)$ & Transitional care program & 30 day all-cause readmission rate $(+)$ & \multirow{4}{*}{ N/A } \\
\hline & & $\begin{array}{c}\mathrm{HF} \\
\sim 79-81 \text { vears }\end{array}$ & APN & $\operatorname{LOS}(-)$ & \\
\hline & & White $\sim 78 \%-84 \%$ & Hospital to home & 60 day direct cost from admission $(-)$ & \\
\hline & & BNP level $>200 \mathrm{ng} / \mathrm{mL}$ & 3 months & $\begin{array}{l}\text { Budget impact analysis (non-statistics, reduced } \\
\text { hospital financial) }\end{array}$ & \\
\hline \multirow{3}{*}{$\begin{array}{l}\text { Simpson (2014) [26] } \\
\text { USA }\end{array}$} & \multirow{3}{*}{$\begin{array}{l}\text { Pre-and post-test (Exp. } \\
\text { only) }\end{array}$} & $\mathrm{N}=263(\mathrm{~N} / \mathrm{A})$ & Nurse-implemented transitional care & \multirow{3}{*}{$\begin{array}{l}30 \text { day readmission rate (non-statistics, } \\
\text { decreased rate) }\end{array}$} & \multirow{3}{*}{$\mathrm{N} / \mathrm{A}$} \\
\hline & & $\begin{array}{c}\mathrm{HF} \\
\mathrm{N} / \mathrm{A}\end{array}$ & $\mathrm{NP}$ & & \\
\hline & & $\begin{array}{l}\mathrm{N} / \mathrm{A} \\
\mathrm{N} / \mathrm{A}\end{array}$ & $\begin{array}{l}\text { Hospital to home } \\
5 \text { months }\end{array}$ & & \\
\hline \multirow{3}{*}{$\begin{array}{l}\text { Yu et al. (2015) [16] } \\
\text { China }\end{array}$} & \multirow{3}{*}{ RCT (Exp. vs. Con.) } & $\mathrm{N}=178(45 \%)$ & $\begin{array}{l}\text { Cardiac nurse-implemented transitional care } \\
\text { Cardiac nurse }\end{array}$ & Event-free survival (-) & \multirow{3}{*}{$\begin{array}{c}\text { Self-care (maintenance, } \\
\text { management, confidence, and } \\
\text { knowledge) }(+) \text { HRQoL }(++)\end{array}$} \\
\hline & & 79 years & Hospital to home & All-cause hospital readmission $(+)$ & \\
\hline & & $\begin{array}{c}\text { N/A } \\
\text { NYHA II/III } \sim 97 \%-98 \%\end{array}$ & 9 months & $\begin{array}{l}9 \text { month mortality }(+) \\
\text { LOS }(+)\end{array}$ & \\
\hline \multirow{3}{*}{$\begin{array}{l}\text { Truong et al. (2015) } \\
\text { [27] USA }\end{array}$} & \multirow{3}{*}{$\begin{array}{l}\text { Cohort study (Exp. vs. } \\
\text { Con.) }\end{array}$} & $\mathrm{N}=632(\sim 49 \%-61 \%)$ & Continuum of Care Network (CCN) program & & \multirow{3}{*}{$\mathrm{N} / \mathrm{A}$} \\
\hline & & $\underset{\sim 68-82 \text { vears }}{\mathrm{HF}}$ & Resident pharmacist & 30 day all-cause hospital readmissions $(++)$ & \\
\hline & & $\begin{array}{l}\text { White } \sim 59 \%-62 \% \\
\text { N/A }\end{array}$ & $\begin{array}{l}\text { Hospital to home } \\
\text { From admission to home after discharge }\end{array}$ & $\begin{array}{l}\text { Compliance with HF-1 at a single community } \\
\text { hospital (++) }\end{array}$ & \\
\hline
\end{tabular}


Table 1. Cont

\begin{tabular}{|c|c|c|c|c|c|}
\hline \multirow{2}{*}{$\begin{array}{l}\text { Author (Year) } \\
\text { Country }\end{array}$} & \multirow{2}{*}{ Study Design } & \multirow{2}{*}{$\begin{array}{l}\text { Sample (Number (Male \%), } \\
\text { Type of HF, Age, Racial } \\
\text { Ethnicity, HF Severity) }\end{array}$} & \multirow{2}{*}{$\begin{array}{l}\text { Contents of Intervention (Intervention, Major } \\
\text { Intervention Provider, Transition Time/Place, } \\
\text { Intervention Duration) }\end{array}$} & \multicolumn{2}{|c|}{ Outcomes (Significance) } \\
\hline & & & & Clinical Outcomes & Patient-Related Outcomes \\
\hline \multirow{5}{*}{$\begin{array}{l}\text { Wong et al. (2016) [28] } \\
\text { China }\end{array}$} & \multirow{5}{*}{ RCT (Exp. vs. Con.) } & $\mathrm{N}=84(52 \%)$ & $\begin{array}{c}\text { Transitional Care Palliative (TCP)-ESHF } \\
\text { program }\end{array}$ & \multirow{5}{*}{ Readmissions at 12 weeks $(++)$} & $\begin{array}{l}\text { Symptom intensity }(+) \\
\text { Functional status }(+)\end{array}$ \\
\hline & & & & & $\mathrm{QoL}(+)$ \\
\hline & & ESHF & NCM & & \\
\hline & & $\begin{array}{l}78 \text { years } \\
\text { N/A }\end{array}$ & Hospital to home & & Satisfaction with care $(+++)$ \\
\hline & & NYHA III/IV 86\% 93\% & 12 weeks & & \\
\hline \multirow{3}{*}{$\begin{array}{l}\text { Ong et al. (2016) [29] } \\
\text { USA }\end{array}$} & \multirow{3}{*}{ RCT (Exp. vs. Con.) } & $\begin{array}{c}\mathrm{N}=1437(54 \%) \\
\text { Decompensated } \mathrm{HF} \\
73 \text { year }\end{array}$ & $\begin{array}{c}\text { Better Effectiveness After Transition-Heart } \\
\text { Failure (BEAT-HF) } \\
\text { Nurses }\end{array}$ & 180 day all-cause readmission $(-)$ & \multirow{3}{*}{$\mathrm{QoL}(+)$} \\
\hline & & $\begin{array}{l}\text { White } \sim 54 \%-55 \% \\
\text { African American } \sim 22 \%-\end{array}$ & Hospital to home & 30 day all-cause readmission $(-)$ & \\
\hline & & $\begin{array}{c}23 \% \\
\text { NYHA III } 64 \%-66 \%\end{array}$ & 180 days & 180 day mortality $(-)$ & \\
\hline \multirow{3}{*}{$\begin{array}{l}\mathrm{O}^{\prime} \text { Connor et al. (2016) } \\
\text { [20] USA }\end{array}$} & \multirow{3}{*}{$\begin{array}{l}\text { Prospective study } \\
\text { (Exp. only) }\end{array}$} & $\mathrm{N}=818(\mathrm{~N} / \mathrm{A})$ & $\begin{array}{l}\text { Telehealth program using the Transitional Care } \\
\text { Model }\end{array}$ & \multirow{3}{*}{$\begin{array}{l}\text { All-cause } 30 \text { day readmission rate (non-statistics, } \\
\text { reduced readmission) }\end{array}$} & \multirow{3}{*}{ N/A } \\
\hline & & $\begin{array}{l}\mathrm{HF} \\
\mathrm{N} / \mathrm{A}\end{array}$ & $\mathrm{RN}$ and telehealth liaisons nurses & & \\
\hline & & $\begin{array}{l}\mathrm{N} / \mathrm{A} \\
\mathrm{N} / \mathrm{A}\end{array}$ & $\begin{array}{l}\text { Hospital to home } \\
\text { Mean } 63 \sim 94 \text { days }\end{array}$ & & \\
\hline \multirow{5}{*}{$\begin{array}{l}\text { Whitaker-Brown et al. } \\
\text { (2017) [17] USA }\end{array}$} & \multirow{5}{*}{$\begin{array}{l}\text { Pre-and post-test (Exp. } \\
\text { only) }\end{array}$} & $\mathrm{N}=50(42 \%)$ & 4-week pilot transition-to-care program & \multirow{5}{*}{$\begin{array}{l}30 \text { day hospital readmission (non-statistics, two } \\
\text { participants were readmitted) }\end{array}$} & \multirow{5}{*}{ HRQoL (+) } \\
\hline & & & Multidisciplinary team & & \\
\hline & & 70 years & & & \\
\hline & & $\begin{array}{c}\text { Caucasian } 83 \% \\
\text { HFrEF (Severe }<20 \% \text { ) }\end{array}$ & Hospital to outpatient setting (Transition clinic) & & \\
\hline & & $\begin{array}{c}\text { HFrEF (Severe }<20 \% \text { ) } 11 \% \\
\text { /HFpEF (Mild } 40 \%-55 \% \text { ) 19\% }\end{array}$ & 4 weeks & & \\
\hline \multirow{4}{*}{$\begin{array}{l}\text { Pacho et al. (2017) [30] } \\
\text { Spain }\end{array}$} & \multirow{4}{*}{$\begin{array}{l}\text { Prospective study } \\
\text { (Exp. vs. Con.) }\end{array}$} & $\mathrm{N}=518(43 \%)$ & APN-directed discharge planning and home & & \multirow{4}{*}{ N/A } \\
\hline & & & follow-up protocol & All-cause 30 day readmission $(+++)$ & \\
\hline & & 82 years & & & \\
\hline & & $\begin{array}{l}\text { N/A } \\
\text { N/A }\end{array}$ & $\begin{array}{l}\text { Hospital to STOP-HE-clinic } \\
30 \text { days }\end{array}$ & HF-related 30 day readmission $(+++)$ & \\
\hline \multirow{4}{*}{$\begin{array}{l}\text { Miller et al. (2017) [31] } \\
\text { USA }\end{array}$} & \multirow{4}{*}{$\begin{array}{l}\text { Prospective study } \\
\text { (Exp. vs. Con.) }\end{array}$} & $\begin{array}{c}\mathrm{N}=\underset{\mathrm{HF}}{4 \mathrm{H}(49 \%)} \\
\end{array}$ & $\begin{array}{l}\text { Multidisciplinary post-acute transitional care } \\
\text { (MDTC) program }\end{array}$ & All-cause readmission rates $(+++)$ & \multirow{4}{*}{ N/A } \\
\hline & & 81 years & Multidisciplinary team & & \\
\hline & & Caucasian $79.5 \%$ & $\begin{array}{l}\text { Hospital to home (connected by Hospital home } \\
\text { care agency) }\end{array}$ & Visit number during the first 2 weeks $(++)$ & \\
\hline & & N/A & 2 weeks & & \\
\hline
\end{tabular}


Table 1. Cont

\begin{tabular}{|c|c|c|c|c|c|}
\hline \multirow{2}{*}{$\begin{array}{l}\text { Author (Year) } \\
\text { Country }\end{array}$} & \multirow{2}{*}{ Study Design } & \multirow{2}{*}{$\begin{array}{l}\text { Sample (Number (Male \%), } \\
\text { Type of HF, Age, Racial } \\
\text { Ethnicity, HF Severity) }\end{array}$} & \multirow{2}{*}{$\begin{array}{c}\text { Contents of Intervention (Intervention, Major } \\
\text { Intervention Provider, Transition Time/Place, } \\
\text { Intervention Duration) }\end{array}$} & \multicolumn{2}{|c|}{ Outcomes (Significance) } \\
\hline & & & & Clinical Outcomes & Patient-Related Outcomes \\
\hline \multirow{4}{*}{$\begin{array}{l}\text { Wong et al. (2017) [32] } \\
\text { China }\end{array}$} & \multirow{4}{*}{ RCT (Exp. vs. Con.) } & $\begin{array}{c}\mathrm{N}=84(\mathrm{~N} / \mathrm{A}) \\
\mathrm{ESHF}\end{array}$ & Transitional Home-based Palliative End-stage & Readmission at 84 days $(+++)$ & QoL (-) \\
\hline & & 76 years & & \multirow{3}{*}{$\begin{array}{c}\text { ER visit at } 84 \text { days }(+) \\
\text { Hospital stay at } 84 \text { days }(+++) \\
\text { Cost (non-statistics, cost-effectiveness } \\
\text { probability) }\end{array}$} & \multirow{3}{*}{$\begin{array}{l}\text { QALY (non-statistics, } 0.0012 \text { at } 28 \\
\text { days/0.0077 at } 84 \text { days) }\end{array}$} \\
\hline & & $\mathrm{N} / \mathrm{A}$ & $\begin{array}{c}\text { NCM } \\
\text { Hospital to home }\end{array}$ & & \\
\hline & & $\mathrm{N} / \mathrm{A}$ & 1 year & & \\
\hline \multirow{3}{*}{$\begin{array}{l}\text { Rezapour-Nasrabad } \\
\text { (2018) [33] Iran }\end{array}$} & \multirow{3}{*}{ RCT (Exp. vs. Con.) } & $\mathrm{N}=168(63 \%)$ & $\begin{array}{c}\text { Transitional care intervention } \\
\text { Liaison nurses }\end{array}$ & \multirow{3}{*}{$\mathrm{N} / \mathrm{A}$} & \multirow{3}{*}{ HRQoL (+) } \\
\hline & & $>65$ years $(30 \%)$ & Hospital to home & & \\
\hline & & N/A & 6 months & & \\
\hline \multirow{4}{*}{$\begin{array}{l}\text { Moye et al. (2018) [34] } \\
\text { USA }\end{array}$} & \multirow{4}{*}{$\begin{array}{l}\text { Cohort study (Exp. vs. } \\
\text { Con.) }\end{array}$} & $\mathrm{N}=177(35 \%)$ & Pharmacy team-led intervention program & $\begin{array}{l}\text { The number of days that elapsed after discharge } \\
\text { to the first readmission }(+)\end{array}$ & \multirow{4}{*}{$\mathrm{N} / \mathrm{A}$} \\
\hline & & $\underset{71 \text { vear }}{\mathrm{HF}}$ & Pharmacist & & \\
\hline & & $\begin{array}{c}71 \text { year } \\
\text { African American 92\% }\end{array}$ & Hospital to home & HF-related readmission (-) & \\
\hline & & $\operatorname{LVEF}(<40 \%) \sim 41 \%-48 \%$ & 10 montins & & \\
\hline \multirow{5}{*}{$\begin{array}{l}\text { Garnier et al. (2018) } \\
\text { [35] Switzerland }\end{array}$} & \multirow{5}{*}{$\begin{array}{l}\text { Cohort study (Exp. vs. } \\
\text { Con.) }\end{array}$} & $\mathrm{N}=1872(\sim 53 \%-54 \%)$ & Multimodal care transition plan & The fraction of days spent for readmissions (-) & \multirow{5}{*}{$\mathrm{N} / \mathrm{A}$} \\
\hline & & & Multidisciplinary team & The rate of readmission $(-)$ & \\
\hline & & $\begin{array}{c}\sim / 6-/ 8 \text { years } \\
\text { N/A }\end{array}$ & Hospital to home & 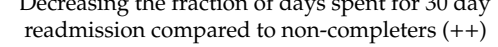 & \\
\hline & & N/A & 13 months & $\begin{array}{l}\text { Decreasing PARE compared with } \\
\text { non-completers }(++)\end{array}$ & \\
\hline & & & & $\begin{array}{l}\text { The rate of PARE decreased } \sim 8.7 \%-9.9 \% \text {, } \\
\text { reaching the adjusted expected range given by } \\
\text { SQLape }{ }^{\circledR}(7.7 \%-9.1 \%)\end{array}$ & \\
\hline \multirow{4}{*}{$\begin{array}{l}\text { Shekarriz-Foumani et } \\
\text { al. (2018) [36] Iran }\end{array}$} & \multirow{4}{*}{ RCT (Exp. vs. Con.) } & $\mathrm{N}=120 \underset{\mathrm{HF}}{(\sim 65 \%-73 \%)}$ & Education and Follow-up after Discharge & Readmission rate $(+)$ & \multirow{4}{*}{ Medication compliance (-) } \\
\hline & & $\sim 65-66$ years & (FAD) program & & \\
\hline & & N/A & Multidisciplinary team & Outpatient visits to physician (-) & \\
\hline & & NYHA III/IV $42 \%-45 \%$ & $\begin{array}{l}\text { Hospital to home } \\
3 \text { months }\end{array}$ & & \\
\hline \multirow{4}{*}{$\begin{array}{l}\text { Reese et al. (2019) [37] } \\
\text { USA }\end{array}$} & \multirow{4}{*}{$\begin{array}{l}\text { Cohort study (Exp. vs. } \\
\text { Con.) }\end{array}$} & & & & \multirow{4}{*}{$\mathrm{N} / \mathrm{A}$} \\
\hline & & Congestive HF & program & 30 day ED or UC visits (-) & \\
\hline & & $\begin{array}{l}74-75 \text { years } \\
\text { nat }\end{array}$ & RN-CM & & \\
\hline & & $\begin{array}{l}\text { White } \sim 90 \%-91 \% \\
\text { N/A }\end{array}$ & $\begin{array}{l}\text { Hospital to home } \\
4 \text { weeks }\end{array}$ & Cost (non-statistics, TCI helps decrease total cost) & \\
\hline
\end{tabular}


Table 1. Cont

\begin{tabular}{|c|c|c|c|c|c|}
\hline \multirow{2}{*}{$\begin{array}{l}\text { Author (Year) } \\
\text { Country }\end{array}$} & \multirow{2}{*}{ Study Design } & \multirow{2}{*}{$\begin{array}{l}\text { Sample (Number (Male \%), } \\
\text { Type of HF, Age, Racial } \\
\text { Ethnicity, HF Severity) }\end{array}$} & \multirow{2}{*}{$\begin{array}{c}\text { Contents of Intervention (Intervention, Major } \\
\text { Intervention Provider, Transition Time/Place, } \\
\text { Intervention Duration) }\end{array}$} & \multicolumn{2}{|c|}{ Outcomes (Significance) } \\
\hline & & & & Clinical Outcomes & Patient-Related Outcomes \\
\hline \multirow{3}{*}{$\begin{array}{l}\text { Van Spall et al. (2019) } \\
\text { [38] Canada }\end{array}$} & \multirow{3}{*}{ RCT (Exp. vs. Con.) } & $\begin{array}{c}\mathrm{N}=2494(50 \%) \\
\mathrm{HF}\end{array}$ & $\begin{array}{l}\text { Patient-Centered Care Transitions in HF } \\
\text { (PACT-HF) service }\end{array}$ & $\begin{array}{l}\text { All-cause readmission at } 30 \text { days }(-), 3 \text { months } \\
\qquad(-)\end{array}$ & $\begin{array}{l}\text { Discharge preparedness at } 6 \text { weeks } \\
(+++)\end{array}$ \\
\hline & & 78 years & $\begin{array}{c}\text { NCM } \\
\text { Hospital to home }\end{array}$ & ED visit at 30 days $(-), 3$ months $(-)$ & Quality of transition at 6 weeks $(+)$ \\
\hline & & N/A & 6 months & Death at 3 months $(-)$ & $\begin{array}{l}\text { QoL at discharge }(+++), 6 \text { weeks } \\
(+), \text { and } 6 \text { months }(+) \\
\text { QALY }(-)\end{array}$ \\
\hline \multirow{4}{*}{$\begin{array}{l}\text { Murphy et al. (2019) } \\
\text { [39] USA }\end{array}$} & \multirow{4}{*}{$\begin{array}{l}\text { Prospective study } \\
\text { (Exp. only) }\end{array}$} & $\mathrm{N}=100(58 \%)$ in $\mathrm{HF}$ & Cardiac Transitions of Care Pilot Program & 30 day readmission rates $(-)$ & \multirow{4}{*}{ N/A } \\
\hline & & $\mathrm{HF}$ & $\begin{array}{l}\text { Multidisciplinary team (physicians, pharmacists, } \\
\text { nurse practitioners, dietitians) }\end{array}$ & $72 \mathrm{~h}$ ED visit rates $(-)$ & \\
\hline & & $\begin{array}{c}68 \text { years } \\
\text { Caucasian } 69 \%\end{array}$ & Hospital to home & 30 day mortality rate $(-)$ & \\
\hline & & N/A & $\begin{array}{c}5 \text { weeks (Inpatient } 1 \text { week and outpatient } 4 \\
\text { weeks) }\end{array}$ & & \\
\hline \multirow{3}{*}{$\begin{array}{l}\text { Plakogiannis et al. } \\
\text { (2019) [18] USA }\end{array}$} & \multirow{3}{*}{$\begin{array}{l}\text { Retrospective cohort } \\
\text { study (Exp. only) }\end{array}$} & $\mathrm{N}=131(57 \%)$ & $\begin{array}{l}\text { Transdisciplinary HF care transition team } \\
\text { (HFCTT) intervention with pharmacy } \\
\text { student-driven postdischarge phone calls }\end{array}$ & \multirow{3}{*}{ Readmission: at 30 days $(++)$ and 90 days $(++)$} & \multirow{3}{*}{ N/A } \\
\hline & & $\begin{array}{c}\mathrm{HF} \\
72 \text { years }\end{array}$ & Pharmacy student (with Multidisciplinary team) & & \\
\hline & & $\begin{array}{l}\text { White } 71 \% \\
\text { HFrEF } 48 \%\end{array}$ & $\begin{array}{l}\text { Hospital to home } \\
\text { Different duration by each patient } \sim 14-60 \text { days }\end{array}$ & & \\
\hline $\begin{array}{l}\text { Wood et al. (2019) [19] } \\
\text { USA }\end{array}$ & $\begin{array}{l}\text { Retrospective cohort } \\
\text { study (Exp. only) }\end{array}$ & $\begin{array}{c}\mathrm{N}=3462(56 \%) \\
\mathrm{HF} \\
72 \text { years } \\
\text { White } 95 \% \text { of } \mathrm{n}=2347 \\
\mathrm{LVEF}(\leq 40 \%) 26 \%\end{array}$ & $\begin{array}{l}\text { Transitions of Care (TOC) Pharmacist Services } \\
\text { Pharmacists and an HF nurse educator } \\
\text { Hospital to home } \\
\text { From admission to } \sim 48-72 \mathrm{~h} \text { after discharge }\end{array}$ & $\begin{array}{l}30 \text { day all-cause readmission (-) } \\
30 \text { day HF readmissions (-) }\end{array}$ & N/A \\
\hline $\begin{array}{l}\text { Neu et al. (2020) [40] } \\
\text { USA }\end{array}$ & $\begin{array}{l}\text { Quasi-experiment } \\
\text { (Exp. vs. Con.) }\end{array}$ & $\begin{array}{c}\mathrm{N}=663(52 \%) \\
\mathrm{HF} \\
\sim 66-69 \text { years } \\
\text { White } 40 \% \text {, Black } 57 \% \\
\text { LVEF }(\leq 40 \%) \sim 51 \%-54 \%\end{array}$ & $\begin{array}{c}\text { Pharmacy-led HF transition of care (TOC) } \\
\text { Pharmacist } \\
\text { Hospital to home } \\
30 \text { days }\end{array}$ & HF 30 day hospital readmission rate $(+)$ & N/A \\
\hline $\begin{array}{l}\text { tistical significanc } \\
\text { ylor Medical Cent } \\
\text { structive pulmona } \\
\text { insitions Teams; H } \\
\text { CM, nurse case ma } \\
\text {; RN-CM, register } \\
\text { spitalized for HF; }\end{array}$ & $\begin{array}{l}\text { disease; C-TraC, } \\
\text { EF, heart failure } 1 \\
\text { ger; NYHA, Nev }\end{array}$ & $\begin{array}{l}1 ;+++P<0.001 ;-P> \\
\text { natriuretic peptide; } \mathrm{C} \\
\text { dinated-transitional car } \\
\text { ced ejection fraction; } \mathrm{H} \\
\mathrm{k} \text { Heart Association; } \mathrm{P} \\
\mathrm{RCT} \text {, randomized cont }\end{array}$ & $\begin{array}{l}\text { 5. AMI, acute myocardial infarction; } \mathrm{AI} \\
\text {, care assessment needs; CHF, chronic h } \\
\text { D, emergency department; EF, ejection } \\
\text { oL, health-related quality of life; LOS, le } \\
\text { hysical therapist; PARE, potentially av } \\
\text { ed trial; STOP-HF-Clinic, STructured m }\end{array}$ & $\begin{array}{l}\mathrm{N} \text {, advanced practice nurses; BHCS, Bay } \\
\text { art failure; CNS, clinical nurse specialis } \\
\text { action; ESHF, end-stage heart failure; } \mathrm{H} \\
\text { gth of stay; LVEF, left ventricle ejection } \\
\text { dable readmission; QALYs, quality-ad } \\
\text { ltidisciplinary outpatient clinic for Old }\end{array}$ & $\begin{array}{l}\text { lor Health Care System; BMCC } \\
\text {; CON, control; COPD, chroni } \\
\text { heart failure; HFCTT, HF Car } \\
\text { fraction; NP, nurse practitione } \\
\text { asted life years; QoL, quality o } \\
\text { and frail Postdischarge patient }\end{array}$ \\
\hline
\end{tabular}


Table 2. Transitional care intervention components pre- and postdischarge.

\begin{tabular}{|c|c|c|}
\hline \multirow{2}{*}{ Author (Year) } & \multicolumn{2}{|c|}{ Transitional Care Intervention Components } \\
\hline & Predischarge Intervention & Postdischarge Intervention \\
\hline Harrison et al. (2002) [21] & $\begin{array}{l}\text { 1. Early assessment after hospital admission; } 2 \text {. Medication } \\
\text { reconciliation; } 3 \text {. Discharge planning patient education }\end{array}$ & $\begin{array}{l}\text { 1. Supportive care for self-management through education or home visit; } \\
\text { 2. Links between hospital and home nurses and patients; } 3 \text {. Balance of } \\
\text { care between the patient and family and professional providers }\end{array}$ \\
\hline Naylor et al. (2004) [22] & 1. Early assessment after hospital admission & 1. Telephone support; 2 . Nurse home visits \\
\hline Williams et al. (2010) [23] & 1. Discharge planning patient education & 1. Follow-up and home visit \\
\hline Barnason et al. (2010) [24] & & 1. Early follow-up after discharge; 2 . Follow-up telephone call \\
\hline Stauffer et al. (2011) [25] & $\begin{array}{l}\text { 1. Screening for eligibility within hospital admission; } 2 . \\
\text { Discharge planning }\end{array}$ & $\begin{array}{l}\text { 1. Early follow-up after discharge; } 2 . \text { Telephone support; } 3 . \text { Nurse home } \\
\text { visits }\end{array}$ \\
\hline Simpson (2014) [26] & 1. Education & 1. Postdischarge telephone contact \\
\hline Yu et al. (2015) [16] & $\begin{array}{l}\text { 1. Appointment schedule before discharge; } 2 \text {. Discharge } \\
\text { planning }\end{array}$ & 1. Home visits; 2. Follow-up telephone call \\
\hline Truong et al. (2015) [27] & $\begin{array}{l}\text { 1. Admission medication review; 2. Daily monitoring; } 3 . \\
\text { Discharge medication review; } 4 \text {. Discharge counseling }\end{array}$ & 1. Early follow-up after discharge \\
\hline Wong et al. (2015) [28] & $\begin{array}{l}\text { 1. Appointment schedule before discharge; } 2 \text {. Discharge } \\
\text { planning }\end{array}$ & 1. Home visit together; 2 . Telephone follow-up \\
\hline Ong et al. (2016) [29] & 1. Predischarge health education & 1. Follow-up telephone call \\
\hline O'Connor et al. (2016) [20] & & $\begin{array}{l}\text { 1. Telemonitoring (personal goal setting, self-monitoring, management of } \\
\text { symptoms, and reporting changes to their physician or care team) }\end{array}$ \\
\hline Whitaker-Brown et al. (2015) [17] & 1. Discharge planning & $\begin{array}{l}\text { 1. Appointment for transition clinic visit (risk assessment, physical } \\
\text { assessment, and evaluation); } 2 \text {. Medication reconciliation; } 3 \text {. Early } \\
\text { follow-up telephone call; } 4 \text {. Providing information related to } \\
\text { rehabilitation, home care, hospice, and/or palliative care }\end{array}$ \\
\hline Pacho et al. (2017) [30] & N/A & $\begin{array}{l}\text { 1. Early postdischarge visit; } 2 \text {. HF nurse education to patient and } \\
\text { caregiver; } 3 \text {. Treatment titration; } 4 \text {. Intravenous medication; } 5 \text {. Early } \\
\text { follow-up via e-notification }\end{array}$ \\
\hline Miller et al. (2017) [31] & N/A & 1. Education and consulting; 2 . Early home visit \\
\hline Wong et al. (2017) [32] & 1. Hospital visit before discharge to introduce the program & 1. Nurse home visit; 2 . Nurse telephone call; 3 . Volunteer social visit \\
\hline Rezapour-Nasrabad (2018) [33] & 1. Nursing care support & 1. Follow-up telephone call \\
\hline
\end{tabular}


Table 2. Cont

\begin{tabular}{|c|c|c|}
\hline \multirow{2}{*}{ Author (Year) } & \multicolumn{2}{|c|}{ Transitional Care Intervention Components } \\
\hline & Predischarge Intervention & Postdischarge Intervention \\
\hline Moye et al. (2018) [34] & $\begin{array}{l}\text { 1. Medication prescript and manage; } 2 \text {. Standard-of-care HF } \\
\text { education program; 3. Medication reconciliation; } 4 \text {. Discharge } \\
\text { planning }\end{array}$ & 1. Postdischarge appointment; 2 . Follow-up phone calls by pharmacy team \\
\hline Garnier et al. (2018) [35] & $\begin{array}{l}\text { 1. Targeted therapeutic education; 2. Caregiver therapeutic } \\
\text { education; } 3 \text {. Medication reconciliation at admission and } \\
\text { discharge; } 4 \text {. Set up of an appointment with the GP; } 5 \text {. } \\
\text { Notification of the GP; } 6 \text {. Community nurse notification; } 7 \text {. } \\
\text { Patient-centered discharge instructions }\end{array}$ & 1. Follow-up telephone call; 2. Telephone support \\
\hline Shekarriz-Foumani et al. (2018) [36] & $\begin{array}{l}\text { 1. Screening for eligibility within hospital admission; } 2 \text {. } \\
\text { Collecting demographic and disease information; } 3 \text {. Explaining } \\
\text { questions to be interviewed on telephone calls }\end{array}$ & $\begin{array}{l}\text { 1. Educating the patients and their guardians immediately after discharge; } \\
\qquad \text { 2. Follow-up telephone call }\end{array}$ \\
\hline Reese et al. (2019) [37] & 1. Discharge planning patient education & 1. Telephone follow-up \\
\hline Van Spall et al. (2019) [38] & $\begin{array}{l}\text { 1. Nurse-led self-care education; } 2 \text {. A structured hospital } \\
\text { discharge summary }\end{array}$ & $\begin{array}{l}\text { 1. Family physician follow-up; } 2 \text {. Postdischarge nurse-led home visits and } \\
\text { heart function clinic care (includes telephone assessment) }\end{array}$ \\
\hline Murphy et al. (2019) [39] & $\begin{array}{l}\text { 1. Admission medication review. 2. Daily monitoring } 3 . \\
\text { Discharge medication review }\end{array}$ & 1. Discharge counseling by telephone; 2 . Postdischarge follow-up \\
\hline Plakogiannis et al. (2019) [18] & 1. Early assessment after hospital admission & $\begin{array}{l}\text { 1. The social worker provided the patient and the caregiver with the } \\
\text { necessary support for a smooth transition into the community; } 2 \text {. } \\
\text { Telephone call by pharmacy student (reviewed the medications, HF } \\
\text { symptoms, and performed a detailed medication reconciliation and } \\
\text { counseling) }\end{array}$ \\
\hline Wood et al. (2019) [19] & $\begin{array}{l}\text { 1. Inpatient medication reconciliation; 2. Medication history } \\
\text { review }\end{array}$ & 1. A follow-up phone call \\
\hline Neu et al. (2020) [40] & $\begin{array}{l}\text { 1. Admission medication reconciliation; } 2 \text {. Discharge medication } \\
\text { reconciliation; } 3 \text {. Patient or caregiver counseling with a focus on } \\
\text { HF medications through verbal and written education materials }\end{array}$ & \\
\hline
\end{tabular}




\section{Results}

\subsection{Characteristics of the Included Studies}

This review included 25 studies regarding transitional care interventions for patients with HF that met our search criteria. In total, 17 of the 25 studies were conducted in North America [17-22, 24-27,29,31,34,37-40], three in Europe [23,30,35], three in China [16,28,32], and two in Iran [33,36]. Thirteen of the 25 studies did not report racial ethnicity $[16,20,21,23,24,26,28,30,32,33,35,36,38]$. The sample size ranged from 38 [24] to 3462 [19], and the average patient age was between 65 [36] and 82 years [27,30]. The percentage of males ranged from 35\% [34] to 97\% [37], but three studies [20,26,32] did not report sex.

\subsection{Type and Severity of HF}

Subjects presented with various HF types: two studies reported on end-stage HF [28,32], three on chronic HF [16,23,33], two on congestive HF [21,37], one on decompensated HF [29], and the remaining 17 on HF [17-20,22,24-27,30,31,34-36,38-40]. To identify HF severity, we considered the New York Heart Association (NYHA) class and left ventricle ejection fraction (LVEF). Only 10 of the 25 studies reported NYHA class or LVEF [16,19,21,22,24,28,29,34,36,40]. One study reported B-type natriuretic peptide level $>200 \mathrm{ng} / \mathrm{mL}$ [25], and two studies reported HF with reduced or preserved ejection fraction [17,18]. The remaining 12 studies [20,23,26,27,30-33,35,37-39] did not report the participants' severity of $\mathrm{HF}$.

\subsection{Methodology of Transitional Care Interventions}

All studies conducted transitional care interventions for patients with HF. Regarding research design, there were 10 RCTs [16,21,22,24,28,29,32,33,36,38], two quasi-experiments [23,40], two pre- and post-tests [17,26], six cohort studies [18,19,27,34,35,37], and five prospective studies $[20,25,30,31,39]$. Although it was necessary to establish controls to verify the effects of transitional care interventions, in six of the 25 studies [17-20,26,39], there was no comparative control group set up to verify the effectiveness of transitional care interventions. In 15 of the 25 studies, the primary intervention providers were nurses [16,20-26,28-30,32,33,37,38], including advanced practical nurses, clinical nurse specialists, research nurses, nurse practitioners, cardiac nurses, registered nurses, nurse case managers, registered nurse case managers, and liaison nurses. A multidisciplinary team served as the primary intervention provider in five studies $[17,31,35,36,39]$. In the remaining five studies $[18,19,27,34,40]$, the major intervention providers were pharmacists or pharmacy students.

Most transitional care intervention supported patients with HF when they directly transitioned from hospital to home. In two studies, patients were transitioned or referred to a transition clinic [17] or structured multidisciplinary outpatient clinic for old and frail postdischarge patients hospitalized for HF [30].

There were different intervention durations, ranging from 2 weeks [18,21,24,31] to 15 months [34]. Five of the 25 studies frequently conducted transitional care interventions for 3 months $[20,22,25,28,36]$. Of the 25 studies, four were conducted for 2 weeks [18,21,24,31], four for 4 weeks [17,30,37,40], and three for 6 months $[29,33,38]$.

\subsection{Outcomes of Transitional Care Intervention}

We attempted to separate the outcomes of the included studies into two categories: clinical and patient-related outcomes. Clinical outcomes included all-cause [16-23,25-32,35-40] and HF-related [19,30,34,40] readmission or rehospitalization, ED or urgent care visit [21,32,37-39], outpatient visit to a physician [36], mortality or death rate [16,29,38,39], event-free survival [16], and LOS [16,23,25]. Patient-related outcomes included self-reported or interviewed measurements such as QoL [21,22,28,29,32,38], HRQoL [16,17,21,24,33], satisfaction [22,23,28], medication adherence [24,36], self-efficacy for HF self-care, self-care, symptom intensity, functional status [16], quality-adjusted life 
years [32,38], and discharge preparedness or quality of transition [38]. Two of the 25 studies [24,33] did not measure clinical outcomes and 13 [18-20,25-27,30,31,34,35,37,39,40] did not measure patient-related outcomes.

\subsection{Effects of Transitional Care Intervention}

The highest frequency of the measured clinical outcomes was for readmission or rehospitalization. Twelve of the 22 studies that measured readmission/rehospitalization showed statistically significant reduction in the rate of readmission/rehospitalization $[16,18,22,25,27,28,30-32,36,37,40]$, although the 10 remaining studies did not $[17,19-21,23,26,29,35,38,39]$. The second highest frequency of clinical outcomes was for ED visit [21,32,37-39] reported in five studies, followed by cost $[22,25,32,37]$ and HF readmission $[19,30,34,40]$ each reported in four studies. Generally, although transitional care interventions tended to reduce cost, the trend was not statistically significant [25,32,37]. Only one study showed a significant reduction of cost [22]. Moreover, transitional care interventions significantly reduced the rate of ED visits [21,32] in two of the five studies as well as the rate of HF readmission $[30,40]$ in two of the four studies. Regarding the aspects of mortality [16,29,39] and LOS [16,23,25], transitional care interventions significantly reduced mortality and LOS in one [16] of the three studies.

The highest frequency of the measured patient-related outcomes was for QoL $[21,22,28,29,32,38]$. In four of the six studies, QoL was significantly betted in the experimental group than in the control group $[22,28,29,38]$. However, five studies reported significantly deteriorated HRQoL [16,17,21,24,33]. Satisfaction with transitional care interventions was significantly greater in the experimental group than in the control group [22,28]. Medication adherence was significantly reduced in one [24] of the two studies [24,36]. Quality-adjusted life years were not significantly changed in two studies [32,38], but discharge preparedness or quality of transition were significantly better in one study [38].

\subsection{Components of Transitional Care Interventions}

The components of transitional care interventions in this study were temporally categorized into two durations: pre- and postdischarge interventions.

\subsubsection{Predischarge Interventions}

A total of 21 of the 25 studies offered predischarge interventions in their transitional care programs [16-19,21-23,25-29,32-40]. In five studies, early assessment or daily monitoring was implemented after hospital admission [18,21,22,27,39]. In two studies, an appointment schedule was planned before discharge to review the required care $[16,28]$. Fourteen studies $[17,21,23,25-29,34,35,37$, 38,40 ] showed that predischarge health education and counseling were an important component of transitional care intervention that includes discharge planning, health education, discharge counseling, and patient-centered discharge instructions. Medication reconciliation upon admission, on discharge, and during hospitalization was described in seven studies $[19,21,27,34,35,39,40]$, and this was also a critical component for improving medication safety in patients with HF. Only one study [35] focused on other components of transitional care intervention, including emotional support by nurses and notification to the general practitioner. Nurses sent a message to the general practitioner regarding patient information before discharge, and community nurses also sent notifications if the patients received assistance from community nursing services.

\subsubsection{Postdischarge Interventions}

A total of 24 of the 25 studies [16-39] emphasized on postdischarge interventions as a main component of transitional care. The patterns of intervention differed across studies, although their nature focused on four main characteristics, namely telephone support, follow-up telephone calls, nurse home visits, and other additional care for outpatients. Three studies $[22,25,26]$ provided telephone support by an advanced practical nurse available 7 days per week and included education and consulting. Ten studies $[16,21-23,25,28,30-32,38]$ offered home visits by HF nurses to monitor and manage signs 
and symptoms after discharge as well as to deliver patient education. One study [32] combined both nurse and volunteer social visits for patients with HF after discharge. Another study [18] involved support from social workers to provide the necessary support and to ensure that the transition services were delivered with comprehensive intervention. Twenty-two studies [16-20,22-30,32-39] reported that early follow-up after discharge and follow-up telephone calls were an advanced intervention in transitional care for patients with HF. This was shown to support the postdischarge education materials and to identify patient's understanding of medication changes and management of HF symptoms since discharge via telemonitoring. Additional care interventions for outpatients after discharge included supportive care for self-management [21], connections between the hospital and community nurses and patients [21], appointments for transition clinic visits to assess risk factors [17], and physical and treatment evaluations of patients [20,38].

\section{Discussion}

In this integrative review, we confirmed the variety and complexity of transitional care interventions that have been proposed. First, we found that transitional care interventions were temporally analyzed over two durations, pre- and postdischarge. Of the 25 studies, 20 [16-19,21-23,25-29,32-39] offered transitional care interventions both before and after discharge. Most transitional care interventions began immediately after hospital admission [16-19,21-23,25-29,32-40] and continued for varying periods after hospital discharge [16-39]. The primary predischarge interventions included discharge planning, patient health education, and counseling $[17,21,23,25-29,34,35,37,38,40]$. Moreover, many interventions involved appointment scheduling before discharge $[16,28]$ as well as explaining or introducing telephone calls or home visits after discharge [32,36]. According to the European Society of Cardiology/American Heart Association guidelines, a multidisciplinary team approach and effective systems of care coordination with special attention to care transitions highlight the importance of preventing readmission or mortality of patients with HF after hospital discharge [41,42]. Transitional care interventions support safe, smooth, and efficient quality transitions and are mainly focused on transition from hospital to home [10]. Unfortunately, despite the need to accurately identify patients' problems and to develop tailored transitional care interventions when patients with HF are admitted to a hospital, early assessment of such patients for transition from hospital to home was presented in only five of the 25 studies $[18,21,22,27,39]$. Many care management interventions for HF have traditionally focused on patients with chronic HF during their outpatient phase [43]. Contrary to this, transitional care interventions managed by a multidisciplinary team have focused on transitions, particularly between the acute and postdischarge phases [17,31,35,36,39]. Transitional care interventions have strengths including early assessment of patients' needs and expected health problems in the home setting before beginning discharge planning for the admission duration. Regarding the assessment of patients' and their families' knowledge and understanding of $\mathrm{HF}$, health providers should begin discharge planning for patients with HF based on their baseline understanding and knowledge of HF that can be corrected before hospital discharge [10].

Second, we analyzed the outcomes of transitional care intervention, namely clinical and patient-related outcomes. The most frequent clinical outcome measure was readmission after hospital discharge. We identified that transitional care intervention significantly reduced the rate of readmission in approximately 55\% (12 of 22 studies) of studies $[16,18,22,25,27,28,30-32,36,37,40]$ in this review. Similar to the present review, some reviews and meta-analyses of patients with congestive HF have reported significantly reduced risks of readmission with transitional care interventions [12,44,45]. Moreover, the most frequent patient-related outcome measures were QoL $[21,22,28,29,32,38]$ and HRQoL $[16,17,21,24,33]$ after hospital discharge. We identified that transitional care intervention significantly improved QoL or HRQoL in more than $90 \%$ of studies in this review $[16,17,21,22,24,28,29,33,38]$. Likewise, another integrative review has reported that transitional care interventions could improve QoL of patients with HF [45]. Transition from hospital to home is a vulnerable time for patients and their families. Therefore, to improve the safety of transition, 
healthcare providers must measure the quality of the transition [46]. Surprisingly, only one [38] of the 25 studies in this review reported discharge preparedness and quality of transition for patients with HF during intervention periods. Although the results of that single, large medical-center study [38] generally support the association between quality of transition (3-Item Care Transitions Measure score) and readmission, they should be interpreted with caution. Of note, the authors recommend that this association should be validated at a large scale, at a hospital level, in future studies [47]. Specially, we identified that transitional care intervention was mainly delivered by nurses in this review, and 12 nurse-led transitional care intervention studies reported significantly improved clinical and patient-related outcomes $[16,21,22,24,25,28-30,32,33,37,38]$. According to a systematic review and meta-analysis, nursing activities such as home visits, case management, and disease management clinics where physicians and cardiac nurses work together with a multidisciplinary HF management team showed significantly decreased all-cause readmission and death [9]. Moreover, in some interventions for heart diseases, the effects of nurse-led transitional care programs appeared excellent $[16,48]$. We confirmed that nurses are the most important healthcare providers of transitional care for patients with HF.

Third, in two large-scale RCTs [29,38], patient-related outcomes but not clinical outcomes were statistically meaningful. This may be because of some specific problems. For instance, in one study performed at 10 hospitals selected via randomization in Canada [38], the eligibility criteria were broad, and patients were included regardless of age, left ventricular function, comorbidities, or prognosis. In another study performed in the United States [29], the better effectiveness after transition-heart failure (BEAT-HF) intervention via telemonitoring did not show significant effects on all-cause readmission within the first 30 or 180 days. These results are related to daily weight changes and physiological signals associated with aggravated symptoms in patients with HF. Moreover, in that study, adequate warnings could not be provided to patients with decompensated HF that recurrence of HF is imminent. Therefore, when conducting a large-sample RCT of transitional care interventions, participant selection criteria should be strictly homogeneous between control and intervention groups, the severity of HF should be assessed based on NYHA class or LVEF, and disease-related conditions should be recorded. Because these factors can affect the risk of readmission or ED visit, they should be taken into account. When providing transitional care, high intensity of interventions and continuous contact of patients with HF with healthcare providers might be crucial to reduce the risk of readmission [44]. Consequently, through meticulous research, transitional care interventions should be tailored according to the severity of disease to achieve the best clinical outcomes in patients with HF.

Finally, HF is a global health concern mostly affecting older people, and research in this setting must consider social, economic, and cultural contexts [49]. Nevertheless, the studies reviewed herein highlight that over $68 \%$ of publications on transitional care interventions for HF are concentrated in North America. In addition, $40 \%$ of studies in North America and Europe did not consider racial ethnicity, which could affect cultural contexts. In these countries, the importance of transitional care intervention has been demonstrated in terms of effectively preventing patients' readmission or ED visits and reducing their mortality. However, in Asia, including Korea, there is still little evidence regarding the effectiveness of transitional care interventions for patients with HF. Thus, research in this area is urgent. Similar to our finding, a previous review has also pointed out the importance of research conducted in various countries [50].

\section{Limitations}

We searched and included only English publications. Therefore, this review synthesizes the best available evidence published in English alone. Moreover, we did not search for all gray literature databases, such as dissertation databases and non-peer reviewed articles, or internet-based search engines (e.g., Google and Google Scholar). Therefore, all relevant research may not have been included in this integrative review. 


\section{Conclusions}

Our findings highlight that transitional care interventions should be developed at the time of patient admission. Transitional care interventions should include early assessment of disease knowledge, with an understanding of what the patients with HF need for self-care at home in order to enhance safe transition from hospital to home. Specifically, before discharge from hospital, the quality of transition should be monitored to confirm that the patient is ready for self-care at home. In addition, we recommend extensive research in Asia, including Korea, to increase the effectiveness of transitional care interventions for patients with HF. We must remember that the role of nurses is very important to realize a good overall multidisciplinary team approach.

Author Contributions: Conceptualization, B.-H.K.; methodology, B.-H.K. and H.M.B.; formal Analysis, B.-H.K. and H.M.B.; investigation and resources, H.M.B. and B.-H.K.; writing-original draft preparation, B.-H.K. and H.M.B.; writing—review and editing, B.-H.K., Y.-J.S., and K.L.; supervision, Y.-J.S. and K.L.; funding acquisition, B.-H.K. All authors have read and agreed to the published version of the manuscript.

Funding: This research was supported by the National Research Foundation of Korea (NRF) grant funded by the Korea government (MSIT) (2019R1I1A2A01058952).

Conflicts of Interest: The authors declare no conflict of interest.

\section{References}

1. Dassanayaka, S.; Jones, S.P. Recent developments in heart failure. Circ. Res. 2015, 117, e58-e63. [CrossRef]

2. von Lueder, T.G.; Agewall, S. The burden of heart failure in the general population: A clearer and more concerning picture. J. Thorac. Dis. 2018, 10, S1934-S1937. [CrossRef]

3. Savarese, G.; Lund, L.H. Global public health burden of heart failure. Card. Fail. Rev. 2017, 3, 7-11. [CrossRef]

4. Mozaffarian, D.; Benjamin Emelia, J.; Go Alan, S.; Arnett Donna, K.; Blaha Michael, J.; Cushman, M.; Das Sandeep, R.; de Ferranti, S.; Després, J.-P.; Fullerton Heather, J.; et al. Heart disease and stroke statistics-2016 Update. Circulation 2016, 133, e38-e360. [CrossRef]

5. Lee, J.H.; Lim, N.-K.; Cho, M.-C.; Park, H.-Y. Epidemiology of heart failure in Korea: Present and future. Korean Circ. J. 2016, 46, 658-664. [CrossRef] [PubMed]

6. Nieminen, M.S.; Dickstein, K.; Fonseca, C.; Serrano, J.M.; Parissis, J.; Fedele, F.; Wikström, G.; Agostoni, P.; Atar, S.; Baholli, L.; et al. The patient perspective: Quality of life in advanced heart failure with frequent hospitalisations. Int. J. Cardiol. 2015, 191, 256-264. [CrossRef] [PubMed]

7. Lewis Eldrin, F.; Claggett Brian, L.; McMurray John, J.V.; Packer, M.; Lefkowitz Martin, P.; Rouleau Jean, L.; Liu, J.; Shi Victor, C.; Zile Michael, R.; Desai Akshay, S.; et al. Health-related quality of life outcomes in PARADIGM-HF. Circ. Heart Fail. 2017, 10, e003430. [CrossRef]

8. Lee, H.; Oh, S.-H.; Cho, H.; Cho, H.-J.; Kang, H.-Y. Prevalence and socio-economic burden of heart failure in an aging society of South Korea. BMC Cardiovasc. Disord. 2016, 16, 215. [CrossRef] [PubMed]

9. Van Spall, H.G.C.; Rahman, T.; Mytton, O.; Ramasundarahettige, C.; Ibrahim, Q.; Kabali, C.; Coppens, M.; Brian Haynes, R.; Connolly, S. Comparative effectiveness of transitional care services in patients discharged from the hospital with heart failure: A systematic review and network meta-analysis. Eur. J. Heart Fail. 2017, 19, 1427-1443. [CrossRef] [PubMed]

10. Albert Nancy, M.; Barnason, S.; Deswal, A.; Hernandez, A.; Kociol, R.; Lee, E.; Paul, S.; Ryan Catherine, J.; White-Williams, C. Transitions of care in heart failure. Circ.: Heart Fail. 2015, 8, 384-409. [CrossRef]

11. Naylor, M.; Sochalski, J. Scaling up: Bringing the transitional care model into the mainstream. Issue Brief (Commonw. Fund) 2010, 103, 1-12. [PubMed]

12. Feltner, C.; Jones, C.D.; Cené, C.W.; Zheng, Z.-J.; Sueta, C.A.; Coker-Schwimmer, E.J.L.; Arvanitis, M.; Lohr, K.N.; Middleton, J.C.; Jonas, D.E. Transitional care interventions to prevent readmissions for persons with heart failure: A systematic review and meta-analysis. Ann. Intern. Med. 2014, 160, 774-784. [CrossRef] [PubMed]

13. Albert, N.M. A systematic review of transitional-care strategies to reduce rehospitalization in patients with heart failure. Heart Lung 2016, 45, 100-113. [CrossRef] [PubMed]

14. Whittemore, R.; Knafl, K. The integrative review: Updated methodology. J. Adv. Nurs. 2005, 52, 546-553. [CrossRef] 
15. Hong, Q.N.; FÀBregues, S.; Bartlett, G.; Boardman, F.; Cargo, M.; Dagenais, P.; Gagnon, M.-P.; Griffiths, F.; Nicolau, B.; O'Cathain, A.; et al. The Mixed Methods Appraisal Tool (MMAT) version 2018 for information professionals and researchers. Educ. Inf. 2018, 34, 1-7. [CrossRef]

16. Yu, D.S.F.; Lee, D.T.F.; Stewart, S.; Thompson, D.R.; Choi, K.-C.; Yu, C.-M. Effect of nurse-implemented transitional care for Chinese individuals with chronic heart failure in Hong Kong: A randomized controlled trial. J. Am. Geriatr. Soc. 2015, 63, 1583-1593. [CrossRef]

17. Whitaker-Brown, C.D.; Woods, S.J.; Cornelius, J.B.; Southard, E.; Gulati, S.K. Improving quality of life and decreasing readmissions in heart failure patients in a multidisciplinary transition-to-care clinic. Heart Lung 2017, 46, 79-84. [CrossRef]

18. Plakogiannis, R.; Mola, A.; Sinha, S.; Stefanidis, A.; Oh, H.; Katz, S. Impact of pharmacy student-driven postdischarge telephone calls on heart failure hospital readmission rates: A pilot program. Hosp. Pharm. 2019, 54, 100-104. [CrossRef]

19. Wood, M.; Sweeney, T.; Trayah, M.; Civalier, M.; McMillian, W. The impact of transitions of care pharmacist services and identification of risk predictors in heart failure readmission. J. Pharm. Pract. 2019. [CrossRef]

20. O'Connor, M.; Asdornwised, U.; Dempsey, M.L.; Huffenberger, A.; Jost, S.; Flynn, D.; Norris, A. Using telehealth to reduce all-cause 30-day hospital readmissions among heart failure patients receiving skilled home health services. Appl. Clin. Inform. 2016, 7, 238-247. [CrossRef]

21. Harrison, M.B.; Browne, G.B.; Roberts, J.; Tugwell, P.; Gafni, A.; Graham, I.D. Quality of life of individuals with heart failure: A randomized trial of the effectiveness of two models of hospital-to-home transition. Med. Care 2002, 40, 271-282. [CrossRef] [PubMed]

22. Naylor, M.D.; Brooten, D.A.; Campbell, R.L.; Maislin, G.; McCauley, K.M.; Schwartz, J.S. Transitional care of older adults hospitalized with heart failure: A randomized, controlled trial. J. Am. Geriatr. Soc. 2004, 52, 675-684. [CrossRef] [PubMed]

23. Williams, G.; Akroyd, K.; Burke, L. Evaluation of the transitional care model in chronic heart failure. Br. J. Nurs. 2010, 19, 1402-1407. [CrossRef] [PubMed]

24. Barnason, S.; Zimmerman, L.; Hertzog, M.; Schulz, P. Pilot testing of a medication self-management transition intervention for heart failure patients. West J. Nurs. Res. 2010, 32, 849-870. [CrossRef] [PubMed]

25. Stauffer, B.D.; Fullerton, C.; Fleming, N.; Ogola, G.; Herrin, J.; Stafford, P.M.; Ballard, D.J. Effectiveness and cost of a transitional care program for heart failure: A prospective study with concurrent controls. Arch. Intern. Med. 2011, 171, 1238-1243. [CrossRef]

26. Simpson, M. A Quality Improvement plan to reduce 30-day readmissions of heart failure patients. J. Nurs. Care Qual. 2014, 29, 280-286. [CrossRef]

27. Truong, J.T.; Backes, A.C. The impact of a continuum of care resident pharmacist on heart failure readmissions and discharge instructions at a community hospital. SAGE Open Med. 2015, 3. [CrossRef]

28. Wong, F.K.Y.; Ng, A.Y.M.; Lee, P.H.; Lam, P.-T.; Ng, J.S.C.; Ng, N.H.Y.; Sham, M.M.K. Effects of a transitional palliative care model on patients with end-stage heart failure: A randomised controlled trial. Heart 2016, 102, 1100-1108. [CrossRef]

29. Ong, M.K.; Romano, P.S.; Edgington, S.; Aronow, H.U.; Auerbach, A.D.; Black, J.T.; De Marco, T.; Escarce, J.J.; Evangelista, L.S.; Hanna, B.; et al. Effectiveness of remote patient monitoring after discharge of hospitalized patients with heart failure: The better effectiveness after transition-heart failure (BEAT-HF) randomized clinical trial. JAMA Intern. Med. 2016, 176, 310-318. [CrossRef]

30. Pacho, C.; Domingo, M.; Núñez, R.; Lupón, J.; Moliner, P.; de Antonio, M.; González, B.; Santesmases, J.; Vela, E.; Tor, J.; et al. Early postdischarge STOP-HF-Clinic reduces 30-day readmissions in old and frail patients with heart failure. Rev. Esp Cardiol. (Engl. Ed.) 2017, 70, 631-638. [CrossRef]

31. Miller, A.; Edenfield, E.E.; Roberto, J.; Erb, J.K. Reduction in re-hospitalization rates utilizing physical therapists within a post-acute transitional care program for home care patients with heart failure. Home Health Care Manag. Pract. 2017, 29, 7-12. [CrossRef]

32. Wong, F.K.Y.; So, C.; Ng, A.Y.M.; Lam, P.-T.; Ng, J.S.C.; Ng, N.H.Y.; Chau, J.; Sham, M.M.K. Cost-effectiveness of a transitional home-based palliative care program for patients with end-stage heart failure. J. Palliat. Med. 2017, 32, 476-484. [CrossRef]

33. Rezapour-Nasrabad, R. Application of transitional care mode in patients with chronic heart disease: A case-controlled intervention study. Rev. Latinoam. Hiperte. 2018, 13, 285-290. 
34. Moye, P.M.; Chu, P.S.; Pounds, T.; Thurston, M.M. Impact of a pharmacy team-led intervention program on the readmission rate of elderly patients with heart failure. Am. J. Health Syst. Pharm. 2018, 75, 183-190. [CrossRef] [PubMed]

35. Garnier, A.; Rouiller, N.; Gachoud, D.; Nachar, C.; Voirol, P.; Griesser, A.-C.; Uhlmann, M.; Waeber, G.; Lamy, O. Effectiveness of a transition plan at discharge of patients hospitalized with heart failure: A before-and-after study. ESC Heart Fail. 2018, 5, 657-667. [CrossRef]

36. Shekarriz-Foumani, R.; Laal, N.; Khosravi, S. The effect of educatoin and follow up after discharge (FAD) on reducing readmission of CHF patients: A randomized controlled trial. Int. J. Health Med. Cur. Res. 2018, 3, 736-743.

37. Reese, R.L.; Clement, S.A.; Syeda, S.; Hawley, C.E.; Gosian, J.S.; Cai, S.; Jensen, L.L.; Kind, A.J.H.; Driver, J.A. Coordinated-transitional care for veterans with heart failure and chronic lung disease. J. Am. Geriatr. Soc. 2019, 67, 1502-1507. [CrossRef]

38. Van Spall, H.G.C.; Lee, S.F.; Xie, F.; Oz, U.E.; Perez, R.; Mitoff, P.R.; Maingi, M.; Tjandrawidjaja, M.C.; Heffernan, M.; Zia, M.I.; et al. Effect of patient-centered transitional care services on clinical outcomes in patients hospitalized for heart failure: The PACT-HF randomized clinical trial. JAMA 2019, 321, 753-761. [CrossRef]

39. Murphy, J.A.; Schroeder, M.N.; Rarus, R.E.; Yakubu, I.; McKee, S.O.P.; Martin, S.J. Implementation of a cardiac transitions of care pilot program: A prospective study of inpatient and outpatient clinical pharmacy services for patients with heart failure exacerbation or acute myocardial infarction. J. Pharm. Pract. 2019, 32, 68-76. [CrossRef]

40. Neu, R.; Leonard, M.A.; Dehoorne, M.L.; Scalia, S.J.; Kale-Pradhan, P.B.; Giuliano, C.A. Impact of pharmacist involvement in heart failure transition of care. Ann. Pharmacother. 2020, 54, 239-246. [CrossRef]

41. Ponikowski, P.; Voors, A.A.; Anker, S.D.; Bueno, H.; Cleland, J.G.F.; Coats, A.J.S.; Falk, V.; González-Juanatey, J.R.; Harjola, V.-P.; Jankowska, E.A.; et al. 2016 ESC Guidelines for the diagnosis and treatment of acute and chronic heart failure: The task force for the diagnosis and treatment of acute and chronic heart failure of the European society of cardiology (ESC) developed with the special contribution of the heart failure association (HFA) of the ESC. Eur. Heart J. 2016, 37, 2129-2200. [CrossRef] [PubMed]

42. Yancy Clyde, W.; Jessup, M.; Bozkurt, B.; Butler, J.; Casey Donald, E.; Drazner Mark, H.; Fonarow Gregg, C.; Geraci Stephen, A.; Horwich, T.; Januzzi James, L.; et al. 2013 ACCF/AHA guideline for the management of heart failure: Executive summary. Circulation 2013, 128, 1810-1852. [CrossRef]

43. Comín-Colet, J.; Enjuanes, C.; Lupón, J.; Cainzos-Achirica, M.; Badosa, N.; Verdú, J.M. Transitions of care between acute and chronic heart failure: Critical steps in the design of a multidisciplinary care model for the prevention of rehospitalization. Rev. Esp Cardiol. (Engl. Ed.) 2016, 69, 951-961. [CrossRef]

44. Vedel, I.; Khanassov, V. Transitional care for patients with congestive heart failure: A systematic review and meta-analysis. Ann. Fam. Med. 2015, 13, 562-571. [CrossRef]

45. Stamp, K.D.; Machado, M.A.; Allen, N.A. Transitional care programs improve outcomes for heart failure patients: An integrative review. J. Cardiovasc. Nurs. 2014, 29, 140-154. [CrossRef]

46. Coleman, E.A.; Mahoney, E.; Parry, C. Assessing the quality of preparation for posthospital care from the patient's perspective: The care transitions measure. Med. Care 2005, 43, 246-255. [CrossRef]

47. Goldstein, J.N.; Hicks, L.S.; Kolm, P.; Weintraub, W.S.; Elliott, D.J. Is the care transitions measure associated with readmission risk? Analysis from a single academic center. J. Gen. Intern. Med. 2016, 31, 732-738. [CrossRef]

48. Zhang, P.; Xing, F.-M.; Li, C.-Z.; Wang, F.-L.; Zhang, X.-L. Effects of a nurse-led transitional care programme on readmission, self-efficacy to implement health-promoting behaviours, functional status and life quality among Chinese patients with coronary artery disease: A randomised controlled trial. J. Clin. Nurs. 2018, 27, 969-979. [CrossRef] 
49. Ziaeian, B.; Fonarow, G.C. Epidemiology and aetiology of heart failure. Nat. Rev. Cardiol. 2016, 13, 368-378. [CrossRef]

50. Boisvert, S.; Proulx-Belhumeur, A.; Gonçalves, N.; Doré, M.; Francoeur, J.; Gallani, M.-C. An integrative literature review on nursing interventions aimed at increasing self-care among heart failure patients. Rev. Lat.-Am Enferm. 2015, 23, 753-768. [CrossRef]

(C) 2020 by the authors. Licensee MDPI, Basel, Switzerland. This article is an open access article distributed under the terms and conditions of the Creative Commons Attribution (CC BY) license (http://creativecommons.org/licenses/by/4.0/). 